\title{
Au risque de l'étranger : un sujet majeur de gouvernance à La Rochelle à la fin du Moyen Âge
}

Mathias Tranchant

\section{OpenEdition}

\section{Journals}

Édition électronique

URL : http://journals.openedition.org/abpo/1001

DOI : $10.4000 / a b p o .1001$

ISBN : 978-2-7535-1517-8

ISSN : 2108-6443

Éditeur

Presses universitaires de Rennes

Édition imprimée

Date de publication : 20 avril 2010

Pagination : $91-108$

ISBN : 978-2-7535-1146-0

ISSN : 0399-0826

\section{Référence électronique}

Mathias Tranchant, «Au risque de l'étranger : un sujet majeur de gouvernance à La Rochelle à la fin du Moyen Âge ", Annales de Bretagne et des Pays de l'Ouest [En ligne], 117-1 | 2010, mis en ligne le 20 avril 2012, consulté le 19 avril 2019. URL : http://journals.openedition.org/abpo/1001 ; DOI : 10.4000/ abpo.1001

Ce document a été généré automatiquement le 19 avril 2019

(c) Presses universitaires de Rennes 


\title{
Au risque de l'étranger : un sujet majeur de gouvernance à La Rochelle à la fin du Moyen Âge
}

\author{
Mathias Tranchant
}

1 Peuplée, au cours des quelques décennies qui suivirent la chute de Châtelaillon en 1131, de migrants venus de toute la façade ponantaise de l'Europe pour y faire fortune, La Rochelle est une ville neuve. Elle est l'une des toutes premières à avoir obtenu en France un statut communal selon les établissements dits de Rouen. Son succès, qui se traduit par la place prééminente qu'elle acquit entre Loire et Gironde dès le XIII ${ }^{\mathrm{e}}$ siècle, elle le dut au remarquable esprit d'entreprise de sa population marchande qui sut prendre part aux déploiements du commerce maritime, ainsi qu'à la clairvoyance de ses gouvernants qui usèrent au mieux, pour le profit de toute la communauté bourgeoise, des moyens politiques dont ils disposaient. Toute entière occupée par les échanges, la ville accueillait une foule cosmopolite, composée de négociants, de facteurs, de marins et d'armateurs, mais aussi d'apprentis et de brassiers employés dans la filière viticole, le plus souvent de passage, parfois établis pour plusieurs mois, voire installés à demeure. L'intensité des transits et brassages, pour profitable qu'elle fût à la santé de l'économie rochelaise, n'était pas sans poser des problèmes ni sans susciter des désordres, et comportait même quelques dangers. Aussi, il revint aux autorités compétentes d'encadrer et de règlementer la venue des étrangers et de leurs biens à La Rochelle et dans sa banlieue.

2 Les motifs de lettres royaux, concédées par Charles VII en faveur des Rochelais en 1446, résument en partie leurs préoccupations en matière de police des étrangers :

«La ville de La Rochelle est assize sur port de mer et est bien notable ville, en laquelle viennent et affluent plusieurs gens, tant de nostre royaume qu'estrangers de diverses nations, [...] pourquoy est besoing et necessité, et aussy pour l'utilité et proffit de la chose publicque d'icelle, mettre et en avoir icelle ville bon nombre, regle, pollice et gouvernement, tant sur le faict des vivres et alliments necessaires a corps humain et qui chacun jour se vendent et distribuent pour la sustentation du 
peuple affluant en ladite ville, que aussy pour tenir nette ladite ville qui est assize

sur port de mer et [...] aisé a corrompre ${ }^{1}$.»

3 Au-delà de ces dispositions, l'étude de la documentation narrative, notariale et normative, et en premier lieu des établissements et des annales de la ville, rend compte de dynamiques fortes autour du pouvoir de police appliqué aux étrangers. Le conseil urbain prit ou fit prendre une somme de décisions à leur égard dont le but fut clairement de faciliter leur venue et leurs activités dans la ville. En dépit des dangers que faisaient peser ennemis, pirates et concurrents, il se montrait résolu à attirer en nombre et durablement des partenaires économiques. La police des étrangers se trouvait donc au cœur de la politique de gestion des risques de la commune. Ce que l'on constate aussi c'est que, dans ce domaine comme dans bien d'autres, l'échevinage rochelais, au contraire de nombre d'institutions urbaines qui virent reculer leurs compétences en la matière au profit d'une administration monarchique grandissante, sut élargir le champ de ses prérogatives. La raison ressortissait à la nécessité de rationaliser la gouvernance des populations allogènes, de prévenir et réduire l'insécurité dont elles pouvaient être porteuses et de limiter les tensions communautaires inhérentes à toute ville ouverte sur le monde.

\section{Une réglementation plus favorable à la venue d'étrangers}

Bien que dépourvue d'une bonne partie de la juridiction appliquée aux étrangers, la municipalité s'appliqua à multiplier les initiatives pour assurer la protection de leurs personnes et de leurs biens ${ }^{2}$.

5 En premier lieu, elle s'évertua à défendre le saufconduit général dont ils bénéficièrent depuis la concession de Louis VIII du 24 août 1224. Par ce moyen, les étrangers à la couronne et leurs biens se trouvaient sous la protection du roi dès lors qu'ils se rendaient à La Rochelle ou en repartaient. Nul ne pouvait exercer contre eux de lettres de marque ${ }^{3}$. De surcroît, les ressortissants d'une puissance ennemie disposaient d'un délai de vingt jours pour se retirer avec leurs marchandises après la publication de la guerre, pourvu qu'ils aient bien acquitté les droits accoutumés ${ }^{4}$. Ce privilège, confirmé une première fois juin 1227 par Louis $\mathrm{IX}^{5}$, revêtait une grande importance au moment des nombreuses tensions et versatilités politiques qui, durant la guerre de Cent Ans, marquèrent les relations internationales. Cette disposition les protégeait également des violences endémiques perpétrées sur mer et dans les ports. Le sauf-conduit royal était de surcroît une condition élémentaire à la stabilité commerciale de la ville. La commune veillait donc au strict respect de cette mesure. Le 7 décembre 1313, sur la requête du corps de ville, et en vertu de son privilège " par lequel lesdites lettres de marque n'y peuvent avoir lieu ", un procès fut engagé à la cour de Poitiers contre des marchands français qui, pourvus de tels documents, avaient fait procéder à la saisie des marchandises de négociants génois à La Rochelle. La cour poitevine donna raison à la municipalité ${ }^{6}$. Les graves troubles de 1346-1347, pendant lesquels Calais tomba aux mains anglaises, décidèrent la commune à saisir Philippe VI, afin qu'il réaffirme le sauf-conduit général, ce qu'il fit au mois d'août de cette dernière année ${ }^{7}$. En 1362, elle dut une nouvelle fois intervenir. Édouard III avait en effet autorisé, malgré sa confirmation des privilèges deux ans plus tôt, la saisie par lettres de marque des biens bretons à La Rochelle, en réparation du pillage de la Catherine de Winchelsea. Le 20 février, devant les remontrances rochelaises, il se ravisa ${ }^{8}$. D'autre part, 
en plus de la protection générale qui était accordée à ses sujets, le souverain anglais leur délivrait des sauf-conduits ponctuels, afin qu'ils puissent venir en toute sécurité en Aunis. En 1364, quatre de ces licences furent octroyées à des marchands de Londres, Mousehole, Yarmouth et Midesummer 9 . On en relève aussi, au cours de cette période, pour leurs collègues de Chichester, Lyme et Exmouth ${ }^{10}$. En 1430, réagissant à l'agression d'un catalan de Béziers par un génois, c'est le parlement de Poitiers qui accorda des saisies compensatoires. Alerté, le corps de ville obtint que sa ville en soit exemptée ${ }^{11}$. Et le 7 décembre, il fit non seulement confirmer son privilège par la cour du Parlement de Paris, mais son application fut étendue aux limites de la banlieue ${ }^{12}$. Cependant, la reconquête française replongea bientôt les gens de mer et les sociétés littorales dans la violence, occasionnant déprédations et saisies arbitraires. Les forains comptaient alors sur l'obtention de sauf-conduits ponctuels pour s'en prémunir. En 1431, Georges de La Trémoïlle se fit payer grassement l'autorisation accordée à des sujets du roi d'Angleterre de mener leurs marchandises à La Rochelle et d'en repartir avec du vin ${ }^{13}$. Encore, le 17 janvier 1464, Jean de Montauban, amiral de France, délivrait sept sauf-conduits, valables une année, à des entrepreneurs anglais, leur permettant de mener leurs affaires dans tous les ports français, sauf dans ceux de la Gironde ${ }^{14}$. Aussi, en novembre 1461, puis le 26 mai 1471, Louis XI réaffirma son sauf-conduit à l'intention de tous les marchands étrangers venant par mer ou par terre à La Rochelle ${ }^{15}$. Surtout, le 27 mai 1472, il étendit davantage encore sa protection.

"Pour améliorer par commerce ladite ville et la condition des habitans d'ycelle", les marchands anglais et autres ennemis du royaume pouvaient désormais « venir en commerce sans fraulde en cette ville et banlieue, toutes et quantes fois que bon leur sembleroyt, quelques guerres qu'il y eust [...], en tels navires, de tel port et équipage qu'il leur plaira, et y charger et descharger telles marchandises et denrées et les vendre, et achepter de tels et à qui ils adviseront ».

6 Il leur fallait toutefois prendre un sauf-conduit auprès des gens du roi ou de son amiral. Et s'ils venaient en groupe de quinze ou vingt marchands, ils devaient aussi obtenir une autorisation d'entrée du maire de la ville ${ }^{16}$. Rien ne permet de juger de l'efficacité réelle de ces mesures. Ce que l'on sait, c'est que les services de l'amirauté, intéressés aux revenus produits par la course, délivraient, trop souvent sans précaution voire abusivement, des lettres de marque qui rendaient inopérantes ces mesures de protection. $\mathrm{Au}$ final, les saisies illicites finissaient devant les juges. Une sentence rendue par la cour du gouvernement de La Rochelle le 9 décembre 1488 témoigne assez bien de cette situation. En vertu du sauf-conduit royal accordé dans la ville aux marchands étrangers, protection qui venait d'être reconfirmée quatre ans plus tôt, elle débouta un Rochelais, Antoine Martin, qui avait fait saisir grâce à une lettre de marque certaines marchandises étrangères ${ }^{17}$.

7 En matière de biens vacants, les allochtones disposaient également de plusieurs garanties. Selon le droit féodal, le détenteur de la puissance publique en était propriétaire. Concrètement, suivant les circonstances ayant conduit à la situation de vacance, il pouvait prétendre à plusieurs droits : le droit d'aubaine, le droit d'épaves et le droit de naufrage. Le premier, qualifié aussi d'aubenage, permettait la saisie des biens d'un étranger décédé dans sa juridiction. Les deux autres, rattachés parfois aux droits de rivage, réglaient la question de tout ce qui était trouvé échoué sur la côte. Si la récupération des biens n'avait pas nécessité l'entremise de sauveteurs et si les éventuels titulaires sinistrés ne s'étaient pas manifestés, le seigneur justicier, faisant valoir son droit d'épaves, se réservait seul leur entière propriété ${ }^{18}$. Dans le cas contraire, le droit de 
naufrage assurait la répartition du produit des biens sauvés entre les différentes parties concernées. Selon la coutume du Poitou, le droit d'épaves s'appliquait après que les officiers seigneuriaux se fussent acquittés d'un certain nombre d'obligations ayant pour but d'assurer la réelle vacance des objets trouvés. Ainsi, ils devaient d'abord informer la population durant trois semaines, par cri public, de la nature des choses échouées et du lieu de leur découverte ; puis quarante jours étaient accordés aux éventuels propriétaires pour se signaler. Ce n'est qu'à l'issue de cette procédure que le seigneur obtenait la pleine propriété de ce que la mer avait rejeté ${ }^{19}$. Mais cette éventualité était rare, parce ce qu'il y avait souvent un détenteur, ou tout du moins un sauveteur, pour prétendre à une partie de cette propriété. C'est sans doute pour cela que le droit d'épave ne figure pas toujours dans les aveux des seigneurs littoraux. C'est aussi pour cette raison qu'il rapportait peu. Quant au droit de naufrage, dit aussi d'aventures ou de bris, il connut un certain assouplissement au cours de la période. Encore au XII ${ }^{\mathrm{e}}$ siècle, entre Loire et Gironde, les biens naufragés revenaient entièrement, au titre du droit d'aubaine, au seigneur côtier ${ }^{20}$. Une inflexion fut donnée à cette exclusivité de sorte que, au siècle suivant, l'exercice du droit de bris donnait lieu désormais à la distribution en trois parts égales du produit des biens récupérés, entre l'autorité banale, les sauveteurs et les marchands sinistrés. Et si une des deux dernières parties était absente, sa portion, considérée alors comme vacante, revenait de fait au seigneur ${ }^{21}$.

Mesurant l'obstacle que constituaient certains de ces droits aux initiatives commerciales étrangères, la municipalité s'y opposa. En ce qui concerne le droit d'épave, les cas de son application étaient rares, et il ne faisait en définitive peser aucune contrainte particulière sur les allochtones. Il ne semble donc pas avoir suscité la désapprobation échevinale. En revanche, le droit d'aubenage, qui autorisait la saisie par les agents royaux des biens d'un négociant étranger décédé au détriment de ses héritiers, y fut, semble-t-il, supprimé en $1374^{22}$. L'étude des privilèges de 1501 précisa au surplus que tous les habitants, «de quelque estat ou condition, pais ou contree et nation", étaient en capacité de tester ${ }^{23}$. Comme partout dans le royaume, résolue à étendre la juridiction du souverain, la prévôté tenta dès qu'elle en avait l'occasion d'appliquer le droit d'aubaine. En mars 1456, Olivier Gansales, originaire de Galice et habitant La Rochelle depuis trente ans, dut faire appel à Charles viI pour être assuré d'en être exempté24. Néanmoins, l'échevinage paraît avoir résisté mieux qu'ailleurs à la généralisation d'un droit d'aubaine royal sur les étrangers ${ }^{25}$. Pour ce qui est du droit de naufrage, sa suppression fut plus précoce mais l'étendue de son application reste difficile à cerner. Dès 1174, Henri II, roi d'Angleterre, avait aboli sur toutes les côtes de l'Aunis et du Poitou l'exercice par les seigneurs côtiers du droit d'aubaine sur les matières naufragées. Ils devaient dès lors les confier à quatre prud'hommes chargés de les garder trois mois, période durant laquelle celui qui en justifiait la propriété récupérait dans leur totalité les biens sauvés qui lui appartenaient ${ }^{26}$. En 1191, Richard Cœur de Lion entérina ce privilège par lequel « toutes personnes quelles qu'elles soient, où elles seront naufragees en mer, facent poursuitte de lever denrees et marchandises sans que les officiers des lieux et terres de son obeissance puissent saisir lesd. biens naufragés et les approprier aud. sieur ${ }^{27} »$. La disposition fut renouvelée et amendée par Philippe VI, sur demande du corps de ville, le 21 mars 1331. Le délai de garde des matières sauvées, "tant deca que dela la mer", et récupérables par leur juste propriétaire, fut étendu à un an et un jour. C'est en ces termes qu'Édouard III le 25 novembre 1360 et Louis XI en novembre 1461, confirmèrent le privilège ${ }^{28}$. Et pourtant, tous les comptes et aveux seigneuriaux témoignent que les châtelains des côtes d'Aunis, 
de Poitou et de Saintonge continuèrent d'exercer dans leur juridiction, jusqu'au début des Temps modernes, le droit de naufrage. Ce dernier leur rapportait des revenus substantiels dont ils n'étaient pas pressés de se dessaisir ${ }^{29}$. Cette contradiction entre décisions royales et réalités locales s'inscrit vraisemblablement dans le processus de lente ingérence de l'État, contre les prérogatives seigneuriales, pour le contrôle du littoral ${ }^{30}$. Aussi, il est probable que l'exemption ne fut en réalité appliquée que le long des rivages relevant directement du roi, ce qui en réduit considérablement le champ d'exercice aux limites strictes du port, à son entrée, ainsi qu'à une partie des côtes situées au nord de la ville. Néanmoins, elle n'était pas sans intérêt, une bonne partie des avaries ayant lieu dans le havre ou à proximité. Ajoutons que ce type de privilège dépendait habituellement d'accords ponctuels, au bénéfice de nationalités particulières ${ }^{31}$. Dans le cas de La Rochelle, la précocité et la généralisation de l'exemption du droit de naufrage à l'ensemble des étrangers constituaient un argument commercial encourageant leur venue.

En matière fiscale, des privilèges particuliers ou généraux étaient aussi recherchés. En 1461, l'échevinage alarmait les autorités royales: «considerans que ladite ville est sur port de mer, et que a l'occasion desdits aydes, les marchans estrangiers et autre y discontinuoient le faict de la marchandise, et que ladicte ville venoit pour la cause dessusdite en grande depopulation et pauvretés ${ }^{32}$. » Dans la compétition que se livraient les marchands des ports du Ponant, notamment sur les secteurs concurrentiels du vin et du sel, le poids des taxes sur les échanges déterminait pour une bonne part leur niveau de compétitivité. C'est pourquoi l'entretien des privilèges fiscaux des forains, ainsi que la modération des devoirs pesant sur le trafic des marchandises, s'imposaient comme des points fondamentaux de la politique commerciale rochelaise. À l'image des protections accordées aux étrangers et à leurs biens, furent établies quelques dispenses générales d'impôts. Elles tendaient pour l'essentiel à favoriser les importations de denrées et d'articles peu ou pas disponibles dans le Centre-Ouest. Dès 1204, Jean sans Terre diminua le droit payé par les forains à leur entrée dans la ville ${ }^{33}$. Par lettres patentes du 6 octobre 1390, Charles VI accorda à tous les négociants venant de l'extérieur du royaume exemption de toute imposition sur la première vente de leurs marchandises à La Rochelle. Un an plus tard, il exonéra pour quelque temps les «marchans estrangiers et regnicoles » de tous les droits d'entrée dans la ville et son port ${ }^{34}$. On trouve renouvellement de sa première disposition en 1461. À cette dernière date fut aussi rappelé que les fournisseurs amenant des céréales dans la ville, par mer ou par terre, étaient déchargés du paiement des douze deniers pour livre. Et pour favoriser les exportations des productions rochelaises, les vins produits à l'intérieur du gouvernement n'étaient pas soumis à la taxe d'un écu par tonneau qui frappait habituellement les crus exportés hors du royaume par des marchands étrangers ${ }^{35}$. La commune restait attentive aux réactions de sa clientèle face aux nouveaux impôts. En 1361, alors qu'Édouard III avait autorisé l'ajout à « la petite imposition » d'une perception de quatre deniers pour livre sur toutes les marchandises vendues et revendues, les Rochelais s'inquiétèrent de voir que certaines transactions produisaient jusqu'à dix-huit deniers pour livre d'impôt. L'année suivante, ils obtinrent donc de lui l'établissement d'un plafond à huit deniers pour livre « affin que ladicte ville soit plus marchande, et que marchans y viennent de toutes parts, et que l'en y aportent toutes manières de vivres et marchandises ${ }^{36} \%$. Quand Charles VI, quelque temps après 1436, instaura la levée d'une nouvelle contribution aux efforts de guerre, ils employèrent un argument similaire pour l'obliger à abandonner cette ambition. Ils lui firent remontrer que, « à l'occasion desdites aydes, les marchans estrangiers et autres y discontinuoient le 
faict de la marchandise ${ }^{37}$ ». Il est vrai que dans ce dernier cas, l'objectif était aussi de se dédouaner d'un impôt qui, par définition, rebutait les bourgeois. Chaque nation bénéficiait de surcroît de privilèges propres, qu'il serait ici trop long d'énumérer et que nous avons déjà eu l'occasion de traiter. Remarquons toutefois que leur délivrance ne fut pas toujours perçue d'un bon œil par la commune, en particulier lorsqu'elle favorisait des communautés concurrentes, comme ce fut le cas pour les Castillans ${ }^{38}$.

Enfin, les étrangers pouvaient être protégés des éventuels abus que des Rochelais, en position de force dans leur ville, leur faisaient subir de temps à autre. En 1478, l'échevinage ordonna que ses bourgeois, vendant leurs marchandises au marché, pratiquassent à l'égard des forains les mêmes tarifs que pour les autochtones ${ }^{39}$. Cependant, ces mesures de protection des étrangers, destinées pour l'essentiel à stimuler leur venue et affermir leur confiance, ne pouvaient égaler en tout point celles dont bénéficiaient les bourgeois, en particulier dans le domaine de la fiscalité. Déterminés à remporter la bataille commerciale que se livraient les grands ports du golfe de Gascogne par l'octroi de multiples privilèges, les Rochelais n'étaient pour autant pas prêts à consentir, dans leur ville, la concurrence des forains. Aussi, la plupart des créations ou exemptions fiscales les privilégiaient-ils davantage.

\section{Les organisations communautaires de forains dans la ville}

Les communautés étrangères établies à La Rochelle disposaient généralement de moyens pour organiser certains aspects de leur propre police. C'était le cas des Castillans qui avaient fait de la capitale aunisienne, non seulement la plaque tournante de leurs initiatives mercantiles vers l'Europe septentrionale, mais aussi le lieu privilégié du règlement des affaires litigieuses survenues entre leurs ressortissants et les autres négociants étrangers. En 1428, alors que de fortes tensions opposaient sur mer Castillans et Flamands, c'est à La Rochelle que fut envisagée la tenue d'une assemblée pour que soient trouvées, avant la fin novembre 1431, des solutions aux conflits. On devait notamment y entendre les plaintes et fixer le montant des indemnités ${ }^{40}$. Au même moment, en 1430, un tribunal permanent y fut institué afin que l'on puisse juger les contestations hispano-bretonnes. Durant les six années (1487-1493) au cours desquelles l'étape de Nantes fut transférée dans la capitale aunisienne, les organes de représentation castillane y furent renforcés de sorte que, malgré son rétablissement ultérieur, un consul continua d'y exercer ses fonctions. La Rochelle fut d'ailleurs choisie en 1497 comme lieu de réunion des maîtres et consuls espagnols attachés à toutes les étapes du royaume de France ${ }^{41}$. Ainsi défendue, la communauté castillane de La Rochelle avait obtenu des conditions de résidence particulièrement avantageuses. Selon une requête de l'échevinage vraisemblablement adressée au cours des années 1487-1493, ils auraient été les seuls étrangers à ne pas participer aux frais de réparation de l'enceinte. De surcroît, ils furent autorisés à percevoir une taxe de six deniers pour livre sur les marchandises qu'ils échangeaient, ainsi qu'un droit de marreage payé par les maîtres des bâtiments ressortissant de leur royaume. Le produit de ces impôts devait, pour partie du moins, être destiné à l'entretien de leurs propres infrastructures et personnels ${ }^{42}$.

Les Flamands, à un moindre degré, avaient également acquis quelques moyens pour diriger et défendre à La Rochelle les membres de leur communauté. C'est en 1462 que 
Louis XI, manifestement soucieux de stimuler les partenariats commerciaux, confirma et élargit les privilèges dont ils pouvaient jouir dans ses ports du Centre-Ouest. D'un point de vue juridique, ils obtinrent reconduction du sauf-conduit royal comme de leurs vieilles exemptions des droits de naufrage et d'aubaine. Ils avaient la possibilité de faire régler les litiges survenant entre leurs ressortissants par les échevins de leurs propres villes. Quant aux procédures d'appels, elles pouvaient être menées devant le tribunal royal le plus proche. Par ce moyen, le roi leur avait garanti la réciprocité des bienveillances dont ses marchands bénéficiaient en Flandre. Surtout, le souverain français leur permettait de réaliser entre eux des transactions en gros sur les denrées qu'ils importaient en Aunis. Ils avaient seulement obligation de les vendre aux non-flamands si ces derniers proposaient un prix équivalent. De cette manière, le roi corrigeait les méthodes d'achat employées notamment par les Rochelais, qui profitaient, afin de faire baisser les prix, du fait que les négociants du nord étaient pressés de vendre leurs articles pour se procurer les liquidités indispensables à leurs acquisitions de vin. Il est certain que cette mesure incitait désormais les sujets du duc de Bourgogne à ne plus être de simples acheteurs, mais aussi des fournisseurs en mesure de trouver à La Rochelle des conditions d'échanges lucratives ${ }^{43}$. Sans constituer une communauté aussi nombreuse que celle des Bretons ou aussi puissante que celle des Castillans, quelques Flamands habitaient durablement dans la ville. Ils y disposaient depuis longtemps de leur propre chapelle, établie dans l'église des Carmes. En 1462, ils furent d'ailleurs autorisés à lever une taxe pour son entretien. Ils obtinrent en même temps la possibilité d'acheter des maisons dans la ville, à condition qu'ils en soient des résidants permanents ${ }^{44}$.

13 L'on sait aussi que jusque vers 1420, la factorerie rochelaise des Hanséates possédait son propre conseil, chargé de transmettre aux ressortissants allemands les décisions du Hansetad et de régler sur place les litiges. En 1421, il fut saisi pour arbitrer un différend entre le Lübeckois Greverode et un confrère de Stralsund ${ }^{45}$. Dans le sillage des exemptions concédées aux Flamands, le Rochelais Guillaume de Combes fut chargé en 1463 de négocier, au nom de Louis XI, les libertés dont les Hanséates étaient spécialement vêtus à La Rochelle. Les termes de l'accord furent ratifiés par le roi l'année suivante, étendus aux ports français de la Manche, puis augmentés par Charles viII par plusieurs lettres patentes de $1483,1487,1489$ et $1490^{46}$. Selon ces documents, ils étaient eux aussi autorisés à acquérir des biens dans le royaume, pouvaient les transmettre à leurs héritiers et étaient assurés en toutes circonstances du sauf-conduit royal.

\section{La gouvernance rochelaise des étrangers : une forme de gestion des risques}

Cependant, c'est en grande partie à l'échevinage que revint le soin, sinon de faire appliquer, tout du moins d'organiser et de réglementer, la venue des étrangers dans sa ville. Quel que fût l'accès qu'ils empruntaient pour entrer dans l'enceinte, ils devaient se conformer à des prescriptions précises. La première d'entre elles n'autorisait le franchissement des portes qu'après s'être soigneusement désarmé. Les navires étaient dégarnis de leurs hunes, " armures et harnoys », et visités par des agents municipaux sur la vase, devant les remparts. Quant aux hommes, ils étaient tenus de déclarer et déposer leurs armes en la maison de l'échevinage ${ }^{47}$. Plusieurs règlements touchaient plus spécifiquement l'accès au port: ils le restreignaient aux seuls vaisseaux chargés de marchandises, l'interdisaient après 17 heures, ou le conditionnaient à l'acquittement de 
diverses taxes. Une fois à l'intérieur de l'enceinte, d'autres obligations s'imposaient aux forains : par exemple celle de délester leurs bateaux dans un lieu approprié, celle de ne pas faire trotter les chevaux dans les rues ou celle d'échanger leurs monnaies nationales pour n'utiliser que des deniers français ${ }^{48}$. Des dispositions réglaient les questions de transbordement. En 1478, il fut interdit aux marchands de vendre et d'acheter des denrées avant qu'elles ne soient descendues à quai et exposées, sous peine de confiscation des biens et d'amende arbitraire. Ils étaient d'autre part tenus de les décharger dans un délai de trois marées ${ }^{49}$. S'agissant des individus, en 1350 , le Conseil statua que ne pouvaient descendre en même temps, de chaque bâtiment, que le maître et vingt marins au maximum ${ }^{50}$. Et s'ils souhaitaient séjourner dans la ville, ils devaient prêter serment de respecter ses établissements ainsi que certaines règles imposées par la législation échevinale. En 1393, la municipalité en allégea la rigueur, notamment à l'égard des Anglais. En temps de trêves, il leur était désormais permis de loger dans La Rochelle, où il leur plaisait, sans que ne leur soit imposé un quelconque logement particulier. Pour seule précaution, la commune exigeait que si des ressortissants d'un même royaume séjournaient en grand nombre dans la ville, ou s'ils se trouvaient concentrés dans certaines maisons, le maire puisse en avoir connaissance et le cas échéant y remédier. En revanche, il ne pouvait profiter de ces circonstances pour percevoir des hôteliers concernés une taxe supplémentaire ${ }^{51}$.

15 La somme de ces mesures de police répondait à une exigence déterminante pour toute communauté urbaine, en particulier pour une ville marchande, située en frontière de mer, en pleine crise de la guerre de Cent Ans : protéger son site, ses institutions et ses habitants des risques que pouvaient représenter l'entrée, la circulation et la résidence de populations étrangères. Les motivations des autorités publiques furent souvent exprimées ainsi : "Laquelle invasion ne menasse d'ennemys qu'on leur ayt sceu faire jacoit ce que ladite ville soit scituee et assise sur la mer en continuel dangier d'estre surprinse $^{52}$." Dans les circonstances politiques et militaires les plus tendues, la règlementation rochelaise pouvait être affermie. On portait alors une attention spéciale aux bâtiments affichant certains pavillons étrangers. En 1378, les gardes de la chaîne ne devaient plus laisser librement entrer dans le port que les sujets du roi de France. Quant aux navires étrangers ou venant d'au-delà de la pointe Saint-Mathieu, leur accueil était dès lors soumis à l'obtention de la licence du maire. En 1399 et 1400, des restrictions furent également prononcées à l'encontre des navires anglais et castillans. Par ailleurs, on veillait sans relâche au contrôle du lieu de résidence des négociants étrangers. Le 12 janvier 1460, l'échevinage obtint de Charles VII le pouvoir d'imposer à tous les hôteliers « de luy rapporter les noms et surnoms, par chasque jour, de ceux qui surviennent loger en leurs maisons ${ }^{53}$ ». Par ces mesures de contrôle d'entrée et de résidence des allochtones, la municipalité entendait se protéger, non seulement des violences qui pouvaient survenir entre les membres de nationalités rivales et les Rochelais, mais aussi d'éventuelles tentatives étrangères de prise de contrôle de la ville, par les armes ou par l'établissement de communautés trop puissantes. Au début des années 1490, la commune s'inquiétait ainsi du nombre de castillans installés à La Rochelle, suite au transfert de la bourse de Nantes: «Item, s'ensuivroit oultre tel inconvenient que les Espaignolz vendroient en si grant habundance en La Rochelle qu'il convendroit que les bons bourgois et habitans nez en icelle ville s'en partissent et laissassent leurs habitacions [et] que les Espaignolz gaignassent la ville, qui seroit tres grant dommaige du Roy du Royaume ${ }^{54}$.» 
16 chaque niveau d'étrangeté au corps des bourgeois. C'est souvent au moyen de ses compétences en matière de police des aliments qu'elle y parvint. La commune maitrisait l'approvisionnement des denrées de base. Elle en contrôlait les tarifs ainsi que les poids et mesures employés dans la ville, ce qui lui permettait de lutter contre les redoutables spéculations et variations des prix ${ }^{55}$. Depuis deux règlements prononcés par Philippe vi en septembre 1338 et février 1339, les bourgeois étaient seuls autorisés à vendre au détail intra muros, disposition que rappela l'échevinage en $1478^{56}$. Cette restriction devait mettre un terme à la concurrence qu'y pratiquaient les marchands de la banlieue, et protéger en particulier l'écoulement des vins rochelais. Ainsi, les nombreuses tavernes établies dans les quartiers de la ville ne pouvaient servir que des vins « creus en la banlieue et en terre de bourgeois ». C'est au maire qu'incombait la délivrance des brevets aux taverniers. De plus, en 1323, il fut exigé de leurs fournisseurs qu'ils se déplacent en personne à l'hôtel de mairie pour prêter le serment que leurs vins étaient bien bourgeois et produits dans la banlieue ${ }^{57}$. Les contrevenants encouraient la confiscation de leurs marchandises et le paiement d'une amende. Mais en 1466, à l'occasion de très nombreuses infractions, la commune eut à réformer cette législation pour circonvenir les pratiques des " paticyers ", « routisseurs » et autres "taverniers publics et ordinaires». Ces derniers vendaient des vins non bourgeois au préjudice des crus bourgeois et contre les statuts communaux. Pour y obvier, l'échevinage interdit aux taverniers de trafiquer du vin en gros dans la ville et rappela aux non-bourgeois leur interdiction d'en pratiquer le débit au détail. Surtout, il chercha à s'assurer par tous les moyens l'origine des vins écoulés dans les tavernes. Les fournisseurs étaient ainsi tenus, avant de se présenter devant le maire pour lui prêter serment, d'obtenir au préalable, du receveur du huitième sur la vente au détail, un brevet certifiant qu'ils vendaient bien des vins bourgeois produits dans la banlieue. Cette disposition fut rappelée par un arrêt de la cour des aides du 7 septembre $1491^{58}$. Il fallait par ailleurs que les taverniers déclarent au maire les nom et prénom de leurs fournisseurs, ainsi que le nombre de tonneaux qu'ils leur avaient achetés. Ces derniers devaient ensuite être frappés de la marque de la ville et de celle du producteur, sur l'un de leurs fonds et sur l'une de leurs douelles, près de la bonde. Si les taverniers vendaient dans leur établissement leurs propres productions, ils étaient tenus chaque année, au moment où ils faisaient acheminer leurs tonneaux dans l'enceinte, de déclarer leur nombre au maire, de jurer la licéité de leur provenance et de les faire marquer comme précédemment décrit. Enfin, il fut prévu que la marque échevinale serait changée annuellement, à la saison des vendanges, et devrait comporter non seulement les armoiries de la ville mais aussi l'écriture du millésime en chiffres romains, "pour monstrer de quelle annee est ledict vin». Cette marque était apposée sous la responsabilité d'un échevin élu tous les ans à cet effet. Il recevait à cette occasion des taverniers, pour son salaire et le paiement du charbon et des autres choses nécessaires, 6 deniers par tonneau marqué. Quant aux contrevenants, ils étaient soumis lors de la première infraction à 60 sous 1 denier d'amende, à la confiscation et la destruction de leurs vins; les fois suivantes, à une amende arbitraire en plus de la confiscation, les sommes ainsi recueillies étant destinées aux fortifications de la ville ${ }^{59}$.

L'activité tonnelière, essentielle pour l'économie viticole rochelaise, reçut, elle aussi, des mesures protectionnistes de la part de la municipalité. L'enjeu était d'importance : 40000 à 45000 tonneaux de vin sortaient annuellement du port de La Rochelle. Leur fabrication faisait vivre tout un pan de l'économie locale qui en tirait des revenus substantiels. Au 
surplus, contrôler cet artisanat, c'était disposer d'un moyen de maîtrise supplémentaire de la filière viticole. Les autorités urbaines cherchèrent dans un premier temps à réserver pour leurs habitants l'exclusivité de la production des futailles dans la ville et sa banlieue. Elles obtinrent ce privilège en février 1347. Toutefois, devant l'opposition des paroisses suburbaines, Philippe VI consentit à annuler le monopole l'année suivante ${ }^{60}$. L'autre objectif de la municipalité fut de déterminer des normes de fabrication des fûts, qui permettraient de les distinguer aisément des tonneaux étrangers à la banlieue, et par conséquent d'identifier et de protéger les vins produits dans l'espace suburbain. La jauge du tonneau rochelais, comprise entre 750 à 850 litres, était inférieure d'une cinquantaine de litres à celle de son principal concurrent, le tonneau bordelais. Cependant, cette faible différence de contenance générait parfois des confusions entre les deux types de futailles ${ }^{61}$. Aussi, les façons du tonneau rochelais étaient également caractéristiques. La largeur, la longueur et l'épaisseur du merrain répondaient à des dimensions spécifiques. Peut-être dès la fin du Moyen Âge, il avait été interdit de cercler les futailles par bandes. Il convenait de les couvrir entièrement de feuillard et d'osier, à l'exception de l'espace d'un pied situé à l'endroit de la bonde. La barre interne qui assurait l'assemblage des fonds du tonneau à ses douves présentait, elle aussi, des caractéristiques spéciales. En dépit de ces spécifications techniques, les fraudeurs, soucieux de vendre leurs vins au meilleur prix, contournaient la règlementation sur les poids et mesures, au dépens des intérêts de la filière viticole rochelaise ${ }^{62}$. Dans les premières années $d u x^{e}$ siècle, un Génois installé à Londres, Richard Garnier, qui chaque année importait en Angleterre quelque 1000 tonneaux de vin de La Rochelle, fut condamné par le lieutenant du gouverneur parce que « fraudulleusement il avoit fait barrer lesditz thonneaux [avec du bois de fayan (hêtre)], a la similitude des vins de Gascougne pour les mieux vandre, en desnomant que lesdits vins estoyent du pais de Gascougne, lesquelz en verité estoyent du pais d'Aulnis, et valloyent mieux pour celle annee que ne faisoyent ceux de Gascougne, et qui eust laissé lesdictz vins ainsy barrez a la similitude de Gascougne, les marchands d'Angleterre fussent plustost tenuz querir les vins clarz oudict pais de Gascougne que audit pais d'Aulnis, parce que les vins clarz d'Aulnis valloyent mieux que ceux de Gascougne ${ }^{63}$ ». En d'autres circonstances, nous l'avons vu, la municipalité avait imposé l'application sur chaque fût de sa marque, ainsi que du millésime du vin qu'il contenait.

L'étrangeté touchait le contenant et, bien entendu, le contenu ${ }^{64}$. Toutefois, en la matière, l'exclusivité rochelaise ne pouvait s'appliquer avec autant de rigueur. En 1229, les crus produits hors de la banlieue n'étant jusque là pas admis dans La Rochelle, un accord passé avec Hugues de Lusignan, appuyé par Isabelle d'Angleterre, autorisa exceptionnellement l'entrée des tonneaux provenant des terres du comte de la Marche jusqu'à la Saint André 65. Ce qui n'était au départ qu'un accord particulier fut bientôt généralisé à l'ensemble des vins d'amont. Louis IX (1230), Philippe III (février 1272), Philippe IV (1295) et Philippe VI (février 1347) élargirent et entérinèrent tour à tour cette disposition qui ne fut ensuite jamais remise en cause. En 1304, Édouard $\mathrm{I}^{\text {er }}$ obtint même ce privilège pour les crus acheminés depuis ses possessions saintongeaises ${ }^{66}$. Au final, tous les vins produits à l'extérieur de la banlieue pouvaient être menés à La Rochelle avant le 30 novembre, sauf si, selon la confirmation de Louis XI de novembre 1461, l'échevinage s'y opposait pour une raison ou une autre ${ }^{67}$. Il était donc préférable pour les marchands rochelais, quitte à souffrir du manque à gagner induit par la présence de crus étrangers, d'accepter la convergence dans leur port d'une partie des productions d'amont au moment de la saison des vins primeurs, plutôt que de voir se développer de nouveaux circuits de distribution. 
Par ailleurs, les années de guerre ou de mauvaises récoltes rendaient parfois nécessaires la venue des vins d'amont. En 1295, par suite de la descente dans la région d'Anglais qui ravagèrent les champs, «les vins de ce païs et de la banlieue avoient esté tellement desgastés qu'il n'y en avoit plus ${ }^{68}$ ». Aussi, il fallut pour ne pas dérouter les acheteurs nordiques importer des vins alentours. En 1325, les gelées ayant été particulièrement sévères en Aunis, les Rochelais se trouvèrent à nouveau dans l'obligation de faire appel en masse aux productions de Saintonge et d'Angoumois. Le 20 juillet, par un accord avec Édouard II, ils s'assurèrent toutefois du caractère exceptionnel de cette mesure, qui ne devait pas porter, à l'avenir, préjudice à leurs privilèges ${ }^{69}$. Une décision similaire dut être prise en juillet $1361^{70}$. Disposant de faveurs fiscales et revendant eux-mêmes une partie des vins de l'arrière-pays, les marchands rochelais conservaient néanmoins dans leur ville un large avantage sur leurs confrères saintongeais et poitevins. La date limite de la SaintAndré leur laissait enfin trois à quatre semaines pour écouler, sans concurrence cette fois-ci, les tonneaux de la banlieue qui n'avaient pas encore trouvé preneurs.

Constatons enfin que la stabilité de l'équilibre trouvé entre vins suburbains et vins d'amont reposait en grande partie sur la discipline des marchands rochelais eux-mêmes. Ceux qui ne possédaient pas leurs propres vignes n'avaient pas nécessairement intérêt à privilégier les crus de la banlieue, et pouvaient aussi espérer quelques bénéfices de la vente en toute période des productions voisines. Les infractions au code du commerce n'étaient d'ailleurs pas rares, surtout après 1453 quand la reconquête française réunit sur un même marché concurrentiel les vins du Centre-Ouest et du Sud-Ouest. Devant l'importance du phénomène - «à la grande destruction du pays et au grand prejudice du public » - l'échevinage dut réagir rapidement. Il était impératif de trouver une parade propre à contrer la pénétration de productions rivales dont la réussite grevait proportionnellement les productions locales. En 1466, les autorités communales prononcèrent ainsi trois règlements portant sur le trafic du vin et du cidre dans la ville. Le premier rappela l'interdiction pesant sur les crus étrangers, qu'ils provinssent $d u$ haut-pays, des îles (Ré et Oléron) ou de Gascogne. Ils ne pouvaient entrer dans La Rochelle depuis la Saint-André jusqu'aux vendanges de l'année suivante, sauf si, chargés avant le 30 novembre, ils n'avaient pu être acheminés à temps dans le port du fait du mauvais temps. Quant aux tonneaux étrangers introduits durant la période autorisée, leurs propriétaires devaient désormais prendre le soin, avant leur déchargement, de déclarer leur nombre au maire et de les faire empreindre de la marque de la ville. Par ce moyen il était possible de les différencier des vins indigènes et d'empêcher ainsi les importations frauduleuses depuis longtemps dénoncées et plus nombreuses que jamais en cette seconde moitié $\mathrm{du} \mathrm{Xv}^{\mathrm{e}}$ siècle. Deux articles supplémentaires réglèrent le cas spécifique des vins de Gascogne et des cidres. La vente en gros ou au détail des premiers fut interdite dès lors qu'ils étaient destinés ensuite à l'exportation. Quant aux " cistres et pommades ", en plein essor à la fin du Moyen Âge, leur entrée fut totalement interdite, quelle que fût la saison ou la qualité de l'importateur ${ }^{71}$. Les négociants et marins étrangers trouvaient dans les nombreuses tavernes rochelaises l'occasion de se restaurer. Ils y buvaient des vins " creus en la banlieue et en terre de bourgeois ", les seuls dont la consommation fût autorisée dans ces établissements. Leur vente au détail était strictement contrôlée par l'échevinage. C'est au maire que revenait la charge d'accorder leurs brevets aux taverniers. De plus, en 1323, il fut exigé de leurs fournisseurs qu'ils se déplacent en personne à l'hôtel de mairie pour prêter le serment que leurs vins étaient bien bourgeois et produits dans la banlieue ${ }^{72}$. Quelques années plus tard, en septembre 1338, Philippe VI 
précisa que les non bourgeois, quelle que fût leur qualité, ne pouvaient vendre des marchandises au détail dans la ville. Il ajouta en février de l'année suivante que les contrevenants risquaient la confiscation de leurs marchandises et le paiement d'une amende.

Approcher la notion d'étrangeté à partir d'une ville telle que La Rochelle offre un point de vue original. Cela tient d'une part à sa situation, maintes fois soulignée par le pouvoir. Charles viconsidérait en 1408 que « la ville de La Rochelle, pour le grand et notable port de mer surquoy elle est assize, et la multitude des marchandz et autres qui chacun jour y asfluent par mer et par terre, a besoing d'estre gouvernee en bonne paix et tranquillité, et lesdits marchands et autres qui la frequentent ${ }^{73} »$. Louis XI évoquait quant à lui en 1462 «notre ville de La Rochelle, qui est assize sur le port de mer en frontiere et continuelle invasion de noz ennemys, ou plusieurs marchans de plusieurs pays et nations estranges viennent et affluent par chacun jour ${ }^{74} »$. Cela tient d'autre part à ses singularités sociales et économiques. Habitée pour l'essentiel de viticulteurs, d'artisans, de marins, de marchands et de juristes, dégagée de toute ingérence et influence ecclésiastique ou seigneuriale, La Rochelle concentrait des activités entièrement dévolues aux échanges. Ici, point d'ordonnancement rigide et figé des populations : cela était contraire aux règles élémentaires du commerce, aux intérêts de la filière viticole et au dynamisme de l'économie maritime. En conséquence, la commune acceptait en conscience d'être exposée sans relâche aux menaces dont pouvait être potentiellement suspecté tout étranger. Elle dut trouver les moyens de gérer au mieux ce risque capital. Il s'agissait bien entendu de protéger la ville et ses bourgeois des attaques, coups de mains, complots et violences armées perpétrés par des puissances ennemies ou de simples délinquants, de contrôler les termes d'un économique incertaine, spécialement dans le domaine du vin, de maitriser les ambitions de communautés allochtones déterminées à s'implanter en masse dans l'enceinte pour y faire fortune. Mais il lui fallait en même temps, pour améliorer sa compétitivité face à la concurrence des places commerciales voisines, protéger les forains, leur garantir des conditions d'accès, de commerce et de résidence toujours plus avantageuses. Ce fut tout l'enjeu de la gouvernance municipale à l'endroit des étrangers.

\section{NOTES DE FIN}

1. BnF, NAF 7285, fo 197-200.

2. Nous reprenons ici des informations déjà publiées dans : TRANCHANT, Mathias, Le Commerce maritime de La Rochelle à la fin du Moyen Âge, Rennes, PUR, 2003, p. 149-154.

3. À ce propos, consulter l'étude de TIMBAL, Pierre-Clément, « Les lettres de marque dans le droit de la France médiévale ", L'étranger. Recueil de la Société Jean Bodin, t. X, $2^{\mathrm{e}}$ partie, 1958, p. 109-138. 
4. Médiathèque La Rochelle, ms 45-46, p. 52.

5. Ibidem, p. 57.

6. BARBot, Amos, Histoire de La Rochelle, Paris/Saintes, éd. Denys-d'Aussy, 1886, p. 123.

7. Ibidem, p. 149.

8. ВARBOT, Amos, Histoire de La Rochelle... op. cit., p. $387 . \mathrm{BnF}, \mathrm{ms}$ fr. $18970, \mathrm{f}^{\circ} 27 \mathrm{v}^{\circ}$.

Médiathèque La Rochelle, ms 45-46, p. 329. Delafosse, Marcel (dir.), Histoire de La Rochelle, Toulouse, 1985 , p. 60-61.

9. Calendar of the Patent Rolls preserved in the Public Record Office, A.D. 1361-1364

(08/05/1364) ; A.D. 1364-1367 (26/10 et 08/12/1364).

10. BARBOT, Amos, Histoire de La Rochelle, op. cit., p. 62.

11. Ibidem, p. 61.

12. Médiathèque La Rochelle, ms 45-46, p. 505.

13. ВАRВOT, Amos, Histoire de La Rochelle, op. cit., p. 62. Calendar of the Patent Rolls, A.D. 1436-1441 (28/12/1439).

14. «Documents sur l'histoire maritime du XV siècle ", éd. Paul Marchegay, Revue des Sociétés Savantes, $6^{\mathrm{e}}$ série, $1875, \mathrm{n}^{\circ} \mathrm{III}$.

15. Médiathèque La Rochelle, ms 45-46, p. 590, 655.

16. BARBOT, Amos, Histoire de La Rochelle, op. cit., p. 409.

17. Médiathèque La Rochelle, ms 45-46, p. 697, 706.

18. Le vieux coustumier de Poictou, Bourges, éd. René Filhol, 1956, p. 249.

19. FAVREAU, Robert, «Les ports de la côte poitevine au XV siècle ", Bulletin philologique et historique, 1962, p. 28.

20. JOURDAN, J. B. Ernest, Éphémérides historiques de La Rochelle, La Rochelle, 1861-1871, rééd. 1979, t. I, p. 173-174.

21. Arch. nat., P $553^{1}, \mathrm{n}^{\circ} 329$ (22/03/1402).

22. Médiathèque La Rochelle, ms 2564-2565, notes manuscrites de Georges Musset. BRONDY, Réjane, Un port de l'Atlantique au Moyen Âge, Lyon, 1969, mémoire de maîtrise inédit, p. 29.

23. BnF, NAF 7285, f $^{\circ} 7$.

24. Arch. nat., JJ 191, $\mathrm{n}^{\circ} 210$.

25. ALTEROCHE, Bernard d', De l'étranger à la seigneurie à l'étranger au royaume, $\mathrm{XI}^{e}-\mathrm{XV}^{e}$ siècle, Paris, 2002, p. 265.

26. JOURDAN, J. B. Ernest, Éphémérides..., op. cit., p. 173-174.

27. Médiathèque La Rochelle, ms 78, A XI. BARBOT, Amos, Histoire de La Rochelle, op. cit., p. 137.

28. BnF, ms. fr. 18970, fo $27 \mathrm{v}^{\circ}$. BARBOT, Amos, Histoire de La Rochelle, op. cit., p. 387. Médiathèque La Rochelle, ms 45-46, p. 257, 328, 598.

29. TRANCHANT, Mathias, Le Commerce maritime..., op. cit., p. 151-152.

30. À ce sujet, voir SARRAZIN, Jean-Luc, «L'État et la seigneurie : le contrôle du littoral poitevin à la fin du Moyen Âge ", Pouvoirs et littoraux du XV $v^{e}$ au XX $X^{e}$ siècle, Actes du Colloque international de Lorient (24, 25, 26 septembre 1998), Rennes, PUR, 2000, p. 29-40.

31. LEVY-BRUHL, Henri, « Le droit de naufrage », Annales du droit commercial et industriel, français, étranger et international, t. 36, 1927, p. 34-53. PASQUIOU, Yves, Du droit d'épave, bris et naufrage, Paris, 1896, p. 48-51.

32. BnF, ms fr. $18970, f^{\circ} 88 r^{\circ}$ et $v^{\circ}$.

33. Médiathèque La Rochelle, ms 45-46, p. 11.

34. BARBOT, Amos, Histoire de La Rochelle, op. cit., p. 246. 
35. Médiathèque La Rochelle, ms 45-46, p. 403, 591, 592.

36. Lettres de rois, reines et autres personnages des cours de France et d'Angleterre depuis Louis VII jusqu'à Henri IV, tirées des archives de Londres par Bréquigny, éd. M. Champollion-Figeac,

t. 2, Paris, 1847, n LXXXVII. FAVREAU, Robert, " La Cession de La Rochelle à l'Angleterre », Actes du $111^{e}$ congrès national des Sociétés Savantes (Poitiers, 1986), Paris, 1988, p. 228-229.

37. BnF, ms fr. $18970, f^{\circ} 88 \mathrm{v}^{\circ}$.

38. TRANCHANT, Mathias, Le Commerce maritime..., op. cit., p. 256-275.

39. Médiathèque La Rochelle, ms 45-46, p. 682-683.

40. Cartulaire de l'Ancienne Estaple de Bruges, éd. L. Gilliodts van Severen, Bruges, 1904-1906, $n^{\circ} 643,648,693$.

41. Archivo General de Simancas, Reg. del Sello, 30 décembre 1497.

42. Bibliothèque Sainte-Geneviève, $\mathrm{ms} 940, \mathrm{f}^{\circ} 238$.

43. Cartulaire de l'Ancienne Estaple, op. cit., $\mathrm{n}^{\circ}$ 1045. CRAEYBECKX, Jan, Un grand commerce d'importation : les vins de France aux anciens Pays-Bas (XIII ${ }^{e}-\mathrm{XVI}^{e}$ siècle), Paris, 1958, p. 123-125.

44. CRAEYBECKX, Jan, Un grand commerce d'importation..., op. cit., p. 123.

45. ABRAHAM-THISSE, Simone, "Les relations hispano-hanséates au Bas Moyen Âge ", En la España medieval, 1992, n 15, p. 259-260.

46. BARBOT, Amos, Histoire de La Rochelle, op. cit., p. 63. WORMS, Émile, Histoire commerciale de la ligue hanséatique, Paris, 1864, p. 226-227.

47. Voir entre autres documents : BnF, ms fr. 18970, fo $28 \mathrm{v}^{\circ}$.

48. TRANCHANT, Mathias, Le Commerce maritime..., op. cit., p. 142-149, 174-175, 181-184.

49. Médiathèque La Rochelle, ms 45-46, p. 682-683.

50. Médiathèque La Rochelle, ibidem, p. 299.

51. Médiathèque La Rochelle, ibid., p. 410.

52. BnF, ms fr 18970, fo $148 \mathrm{v}^{\circ}$.

53. TRANCHANT, Mathias, Le Commerce maritime..., op. cit., p. 178-180. BARBOT, Amos, Histoire de La Rochelle, op. cit., p. 336.

54. Bibliothèque Sainte-Geneviève, ms. 940, fo 238.

55. TRANCHANT, Mathias, Le Commerce maritime..., op. cit., p. 193-195.

56. Arch. nat, JJ 71, n 177. ВАRBOT, Amos, Histoire de La Rochelle, op. cit., p. 142-143.

Médiathèque La Rochelle, ms 45-46, p. 276, 682-683.

57. BnF, ms fr. 16908, f $246 \mathrm{r}^{\circ}$ et $\mathrm{v}^{\circ}$; d'après FAVREAU, Robert, « La Rochelle, port français sur l'Atlantique au XIII ${ }^{\mathrm{e}}$ siècle ", L'Europe et l'Océan au Moyen Âge, Paris, 1988, p. 56. BARBOT, Amos, Histoire de La Rochelle, op. cit., p. 142-143. Médiathèque La Rochelle, ms 45-46, p. 243, 276.

58. BARBOT, Amos, Histoire de La Rochelle, op. cit., p. 445.

59. Médiathèque La Rochelle, ms 45-46, p. 619-621.

60. Arch. nat, $\mathrm{X}^{1 \mathrm{C}} 4, \mathrm{n}^{\circ} 200$.

61. TRANCHANT, Mathias, Le Commerce maritime..., op. cit., p. 110-112. La Coutume de Royan au Moyen Âge, éd. Georges Musset, Saintes, 1904, nº VII.

62. RENOUARD, Yves, « Le grand commerce des vins de Gascogne au Moyen Âge ", Études d'histoire médiévale, Paris, 1968, p. 281. BnF, ms fr. 16906, fo $126 \mathrm{r}^{\circ}$ et $\mathrm{v}^{\circ}$.

63. BnF, $\mathrm{ms}$ fr. $16906, \mathrm{f}^{\circ} 126 \mathrm{r}^{\circ}$ et $\mathrm{v}^{\circ}$.

64. Nous reprenons ici des informations déjà publiées dans : TRANCHANT, Mathias, Le Commerce maritime..., op. cit., p. 114-119.

65. Médiathèque La Rochelle, ms 45-46, p. 61. BARBOT, Amos, Histoire de La Rochelle, op. cit., p. 80-82. BnF, NAF $7285, f^{\circ} 43 r^{\circ}$ et $v^{\circ}$. 
66. Médiathèque La Rochelle, ms 45-46, p. 135, 183, 202. Arch. Nat., JJ 77, nº 68.

67. Médiathèque La Rochelle, ms 45-46, p. 597.

68. BARBOT, Amos, Histoire de La Rochelle, op. cit., p. 108-112.

69. Ibidem, p. 133-134.

70. FAVREAU, Robert, « La Cession de La Rochelle à l'Angleterre », Actes du $111^{e}$ congrès

national des Sociétés Savantes (Poitiers, 1986), Paris, 1988, p. 228. Aunis, Saintonge et Angoumois, op. cit., pièce $n^{\circ} 11$ (20 juillet 1361). HUET, Étienne, Commentaires sur la coutume de La Rochelle et pays d'Aunix, La Rochelle, 1688, p. 709. Baudouin donne, quant à lui, la date du 20 juillet 1365. Médiathèque La Rochelle, ms 45-46, p. 331.

71. Médiathèque La Rochelle, ms 45-46, p. 621-622.

72. BnF, ms fr. 16908, fo $246 \mathrm{r}^{\circ}$ et $\mathrm{v}^{\circ}$. BARBOT, Amos, Histoire de La Rochelle, op. cit., p. 142-143. Médiathèque La Rochelle, ms 45-46, p. 243, 276.

73. BnF, ms fr. $16906, f^{\circ} 118$.

74. BnF, ms fr. $18970, f^{\circ} 131$.

\section{RÉSUMÉS}

Approcher la notion d'étrangeté à partir d'une ville telle que La Rochelle offre un point de vue original. Cela tient d'une part à sa situation frontalière. Cela tient d'autre part à ses singularités sociales et économiques. Composée pour l'essentiel de viticulteurs, d'artisans, de marins, de marchands et de juristes, largement dégagée des ingérences et influences ecclésiastiques ou seigneuriales, La Rochelle concentrait des activités entièrement dévolues aux échanges. Ici, point d'ordonnancement rigide et figé des populations : cela était contraire aux règles élémentaires du commerce, aux intérêts de la filière viticole et au dynamisme de l'économie maritime. En conséquence, la commune acceptait en conscience d'être exposée sans relâche aux menaces dont pouvaient être suspectés les étrangers. Elle était bien-sûr tenue de protéger la ville, ses bourgeois et leurs intérêts des attaques, des déprédations ou des implantations perpétrées par des puissances étrangères. Mais il lui fallait en même temps, pour améliorer sa compétitivité face à la concurrence des places commerciales voisines, protéger les forains, leur garantir des conditions d'accès, de commerce et de résidence toujours plus avantageuses. Ce fut tout l'enjeu de la gouvernance municipale à l'endroit des étrangers.

Approaching the concept of foreignness from a city like La Rochelle offers an original point of view. This is partly due to its border situation. This is partly due to its social and economic singularities. Composed essentially of vine growers, artisans or seamen, merchants and lawyers, largely free from interferences and influences ecclesiastical or stately, La Rochelle concentrated activities fully devoted to trade. Here, no fixed and rigid ordering of people : this was contrary to the basic rules of trade, the interests of the wine industry and the dynamism of the maritime economy. Consequently, the municipality agreed in conscience to be exposed tirelessly to threats of which foreigners could be suspected. It was of course bound to protect the city, its citizens and their interests against attacks, depredations or settlements perpetrated by foreign powers. But, at the same time, in order to improve its competitiveness with neighbouring commercial centres, it must protect the foreigners and guarantee them ever more advantageous conditions of access, trade and residence. This was the issue of the municipal ruling as regarded foreigners. 
INDEX

Index chronologique : Moyen Âge

Thèmes : La Rochelle

\section{AUTEUR}

\section{MATHIAS TRANCHANT}

Maître de conférences d'histoire médiévale université de La Rochelle Centre de Recherche en Histoire Internationale et Atlantique - EA 1163 